\title{
Icariin promotes stable chondrogenic differentiation of bone marrow mesenchymal stem cells in self-assembling peptide nanofiber hydrogel scaffolds
}

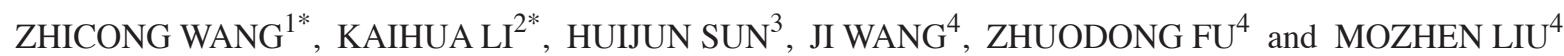 \\ ${ }^{1}$ Department of Orthopedic Surgery, People's Hospital of Deyang City, Deyang, Sichuan 618000; \\ ${ }^{2}$ Department of Orthopedic Surgery, General Hospital of Fengfeng Group, Handan, Hebei 056200; \\ ${ }^{3}$ Department of Clinical Pharmacology, Dalian Medical University, Dalian, Liaoning 116044; ${ }^{4}$ Department of \\ Orthopedic Surgery, The First Affiliated Hospital of Dalian Medical University, Dalian, Liaoning 116011, P.R. China
}

Received January 12, 2016; Accepted December 22, 2016

DOI: $10.3892 / \mathrm{mmr} .2018 .8913$

\begin{abstract}
Icariin, a traditional Chinese medicine, has previously been demonstrated to promote chondrogenesis of bone marrow mesenchymal stem cells (BMSCs) in traditional 2D cell culture. The present study investigated whether icariin has the potential to promote stable chondrogenic differentiation of BMSCs without hypertrophy in a 3D microenvironment. BMSCs were cultivated in a self-assembling peptide nanofiber hydrogel scaffold in chondrogenic medium for 3 weeks. Icariin was added to the medium throughout the culture period at concentrations of $1 \times 10^{-6} \mathrm{M}$. Chondrogenic differentiation markers, including collagen II and SRY-type high mobility group box 9 (SOX9) were detected by immunofluorescence, reverse transcription-quantitative polymerase chain reaction and toluidine blue staining. Hypertrophic differentiation was further assessed by detecting collagen $\mathrm{X}$ and collagen I gene expression levels and alkaline phosphatase activity. The results demonstrated that icariin significantly enhanced cartilage extracellular matrix synthesis and gene expression levels of collagen II and SOX9, and additionally promoted more chondrocyte-like rounded morphology in BMSCs. Furthermore, chondrogenic medium led to hypertrophic differentiation via upregulation of collagen $X$ and collagen I gene expression levels and alkaline phosphatase activity, which was not potentiated by icariin. In conclusion, these results suggested
\end{abstract}

Correspondence to: Professor Mozhen Liu, Department of Orthopedic Surgery, The First Affiliated Hospital of Dalian Medical University, 222 Zhongshan Road, Dalian, Liaoning 116011, P.R. China

E-mail: mozhenliu@hotmail.com

*Contributed equally

Key words: icariin, bone marrow mesenchymal stem cells, chondrogenic differentiation, hypertrophic differentiation, self-assembled peptide nanofiber scaffold that icariin treatment may promote chondrogenic differentiation of BMSCs, and inhibit the side effect of growth factor activity, thus preventing further hypertrophic differentiation. Therefore, icariin may be a potential compound for cartilage tissue engineering.

\section{Introduction}

Articular cartilage damage is one of the most common diseases seen in the clinic, and has been a challenge in orthopedic medicine due to the poor self-healing ability of cartilage tissue (1). Severe cartilage damage, initially caused by degeneration or trauma, may lead to osteoarthritis (OA) and subsequently cause joint pain and disability, creating a significant disease burden worldwide. Although various conservative interventions have been developed for the treatment of early OA, including oral nonsteroidal anti-inflammatory drugs and intra-articular hyaluronic acid injections, they only temporarily alleviate pain. For severe degenerative joint diseases, surgical arthroplasty is an effective treatment; however, it has significant postoperative complications. Due to the potential of chondrogenic differentiation and extensive proliferation of bone marrow mesenchymal stem cells (BMSCs), cartilage tissue engineering is currently considered to be one of the most promising techniques for the treatment of OA (1). To induce chondrogenic differentiation of BMSCs, numerous growth factors are applied, which have previously been demonstrated to enhance chondrogenesis and promote formation of cartilage-like tissue (2-4). However, growth factors inevitably upregulate the expression levels of hypertrophic differentiation markers, including collagen $\mathrm{x}$, matrix metalloproteinase 13 and alkaline phosphatase (ALP), and functionally contribute to calcification (2-5). Additional side effects of growth factor treatment limit their clinical use, including expense, rapid degradation and loss of activity (6-8). One strategy for addressing these issues is to investigate safe and low-cost drugs that may substitute or cooperate with growth factors to promote and maintain stable chondrogenic differentiation without hypertrophy $(6,7)$.

Herb Epimedium (HEP) is a traditional Chinese herb and is widely used to treat osteoporosis and OA in China, Japan 
and Korea (8). Icariin $\left(\mathrm{C}_{33} \mathrm{H}_{40} \mathrm{O}_{15}\right.$; molecular weight, 676.65) is the primary pharmacologically active compound of HEP. When chondrocytes were used as seed cells, icariin increased cartilage extracellular matrix (ECM) synthesis, suppressed ECM degradation, enhanced cartilage-specific gene expression including collagen II, aggrecan and SRY-type high mobility group box 9 (SOX9) in vitro, and additionally improved the repair of cartilage defects and prevented cartilage degradation in vivo $(6,7,9,10)$. The application of chondrocytes in cartilage tissue engineering is prevalent, yet it faces numerous challenges, including chondrocyte dedifferentiation, donor site morbidity and limited sources for harvesting cartilage tissue (1). In an attempt to overcome these challenges, our recent study cultivated BMSCs with chondrogenic medium containing icariin in monolayer culture for 14 days, which is a traditional two-dimensional (2D) cell culture, and demonstrated that icariin promoted directed chondrogenesis of BMSCs and had no effect on hypertrophic differentiation (11). However, chondrogenic potential of stem cells or chondrocytes cultured in a three-dimensional (3D) microenvironment is different from 2D culture $(12,13)$. Thus, icariin may be a potential accelerator for cartilage tissue engineering; however, its effects on BMSCs in a 3D microenvironment require further investigation to elucidate its clinical application.

To culture BMSCs in a 3D microenvironment, the scaffold is a critical component and should mimic the structural and functional properties of the native ECM to accommodate cells, and additionally facilitate cell migration, proliferation and differentiation. Self-assembling peptides are a relatively novel class of molecules that have the ability to form stable nanofiber hydrogels upon exposure to physiological $\mathrm{pH}$ and ionic strength. The self-assembling peptide nanofiber hydrogel scaffold exhibits excellent biocompatibility, supports the chondrocyte phenotype (14), promotes BMSCs proliferation and chondrogenic differentiation in vitro (15-18), and stimulates cartilage regeneration or improves clinical symptoms in vivo $(19,20)$. Therefore, a self-assembling peptide hydrogel scaffold was considered as an ideal scaffold for 3D cell culture and cartilage tissue engineering (21).

The present study extended our previous investigations to observe the effect of icariin on chondrogenic differentiation of BMSCs in a self-assembling peptide nanofiber hydrogel scaffold. To the best of our knowledge, this is the first report of its kind. These findings demonstrated that icariin promotes BMSCs chondrogenesis; however, has no significant effect on hypertrophic differentiation in a 3D microenvironment.

\section{Materials and methods}

Cell culture of rat BMSCs. OriCell ${ }^{\mathrm{TM}}$ Sprague-Dawley rat BMSCs (catalog no. RASMX-01001) were purchased from Cyagen Biosciences Inc. (Guangzhou, China). Cell viability, sterility, purity, proliferation and differentiation ability were tested by the company, which revealed that cells were highly positive for the specific mesenchymal markers CD29 (83.99\%), CD44 (99.69\%) and CD90 (95.05\%), and negative for the hematopoietic cell-surface markers CD34 (0.62\%), CD45 (0.28\%), and CD11b (4.25\%). To verify pluripotency, BMSCs were able to differentiate into osteoblasts, chondrocytes and adipocytes. Cells were thawed at $37^{\circ} \mathrm{C}$ in a water bath and resuspended in low glucose Dulbecco's modified Eagle's medium (LG-DMEM) supplemented with $10 \%$ fetal bovine serum, $10 \mathrm{U} / \mathrm{ml}$ penicillin $\mathrm{G}$ and $10 \mathrm{mg} / \mathrm{ml}$ streptomycin, all purchased from Hyclone (GE Healthcare Life Sciences, Logan, UT, USA). The cell suspension was subsequently plated into T25 flasks and incubated in a 5\% carbon dioxide humidified incubator at $37^{\circ} \mathrm{C}$. Upon achieving $80-90 \%$ confluence, cells were treated with $0.25 \%$ trypsin $/ 1 \mathrm{mM}$ ethylenediaminetetraacetic acid (Gibco; Thermo Fisher Scientific, Inc., Waltham, MA, USA) for 3-5 min. In order to harvest an adequate number of cells, cells at passage 5 to 7 were used in this study.

Hydrogel encapsulation and $3 D$ culture. A BeaverNano ${ }^{\mathrm{TM}}$ 3D hydrogel scaffold (catalog no. P0030105; Cyagen Biosciences Inc.), which was a self-assembling peptide nanofiber scaffold with pore sizes between 50 and $200 \mathrm{~nm}$, was selected because it had 3D nanofiber structures similar to natural cartilage ECM. The molecular mode (Ac-RADARADARADARADA-CONH${ }_{2}$ ), microstructure (visualized by scanning electron microscopy and atomic force microscopy) and preparation of the hydrogel scaffold are detailed by the manufacturer (Beaver Nano-Technologies Co., Ltd., Suzhou, China), and a $0.25 \%$ concentration was applied in the present study. Briefly, $1.0 \%$ (w/v) hydrogel stock solution was mixed with an equal volume of $20.0 \%$ (w/v) sucrose solution. Following trypsinization, BMSCs were centrifuged at $400 \times \mathrm{g}$ for $5 \mathrm{~min}$ at $4^{\circ} \mathrm{C}$, resuspended in $10.0 \%(\mathrm{w} / \mathrm{v})$ sucrose solution, and quickly encapsulated in an equal volume of $0.50 \%(\mathrm{w} / \mathrm{v})$ concentration peptide scaffolds to make a final concentration of $1.0 \times 10^{7}$ cells $/ \mathrm{ml}$. The cell density was selected to match a previous study (15). The cell/hydrogel mixture $(300 \mu \mathrm{l})$ was immediately dropped onto the bottom of each cell culture well $\left(200 \mathrm{~mm}^{2} /\right.$ hole; EMD Millipore, Billerica, MA, USA) and allowed to form a layer $\sim 1.50-\mathrm{mm}$ thick. LG-DMEM medium (500 $\mu \mathrm{l})$ was added to the top of the scaffold to induce self-assembly. The cell/hydrogel scaffolds were incubated in a humidified atmosphere with $5 \% \mathrm{CO}_{2}$ at $37^{\circ} \mathrm{C}$.

Chondrogenic differentiation. Following self-assembly, the cell/hydrogel scaffolds were cultured in control medium without chondrogenic supplements, or chondrogenic medium (catalog no. GUXMX-90041, Cyagen Biosciences Inc.) containing $10 \mathrm{ng} / \mathrm{ml}$ transforming growth factor (TGF)- $\beta 3$ in the presence or absence of $1 \times 10^{-6} \mathrm{M}$ icariin (labeled control, TGF- $\beta 3$ + icariin or TGF- $\beta 3$ groups, respectively). Icariin (purity: 99\%) was purchased from National Institute for the Control of Pharmaceutical and Biological Products (Beijing, China). OriCell ${ }^{\text {Th }}$ MSC chondrogenic differentiation medium contained $0.1 \mu \mathrm{M}$ dexamethasone, $50 \mu \mathrm{g} / \mathrm{ml}$ ascorbate, $1 \%$ insulin-transferrin-selenium cell culture supplement, $100 \mu \mathrm{g} / \mathrm{ml}$ sodium pyruvate, $40 \mu \mathrm{g} / \mathrm{ml}$ proline and $10 \mathrm{ng} / \mathrm{ml}$ TGF- $\beta 3$. In the TGF- $\beta 3$ + icariin group, icariin was dissolved in dimethyl sulfoxide and subsequently added to chondrogenic differentiation medium at concentrations of $1 \times 10^{-6} \mathrm{M}$, according to our preliminary experiments (11). The culture medium was replaced every other day, and the morphology of BMSCs was observed under a CKX41 inverted microscope (Olympus Corporation, Tokyo, Japan). At each time point (days 7, 14 and 21), the scaffold, cell lysis and culture medium 
were harvested for further experiments. All experiments were performed at least three times.

Histology and immunohistochemistry. Cell/hydrogel scaffolds were fixed in $4 \%$ paraformaldehyde and permeabilized in PBS containing $0.1 \%$ Triton X-100 at room temperature for 30 min. Following blocking with $5 \%$ bovine serum albumin (GE Healthcare Life Sciences) for $2 \mathrm{~h}$, samples were treated with the following primary antibodies: Rabbit anti-collagen II (catalog no. GTX20300; 1:100; GeneTex, Inc., Irvine, CA, USA), rabbit anti-SOX9 (catalog no. ab71762; 1:200; Abcam, Cambridge, UK) or mouse anti-collagen $\mathrm{x}$ (catalog no. ab49945; 1:200; Abcam) at $4^{\circ} \mathrm{C}$ overnight, washed three times with PBS, and subsequently incubated with cy3-labeled goat anti-rabbit fluorescent secondary antibody (catalog no. A0516; 1:1,000; Beyotime Institute of Biotechnology, Shanghai, China) or cy3-labeled goat anti-mouse fluorescent secondary antibody (catalog no. A0521; 1:1,000; Beyotime Institute of Biotechnology) for $2 \mathrm{~h}$ at room temperature in the dark. Cell nuclei were stained with 4, 6-diamidion-2-phenylindole (catalog no. C1006; 1:1,000; Beyotime Institute of Biotechnology) for $3 \mathrm{~min}$. Subsequently, stained samples were imaged under a Leica DM4000 B fluorescence microscope (Leica Microsystems GmbH, Wetzlar, Germany). Additional samples were washed three times with PBS and stained for sulfated proteoglycans with toluidine blue dye solution (0.50\%; Abcam) for $5 \mathrm{~min}$, and subsequently observed under an inverted microscope (Olympus Corporation, Tokyo, Japan).

Cell abstraction from hydrogel scaffold and reverse transcription-quantitative polymerase chain reaction (RT-qPCR). At days 7, 14 and 21, BMSCs from hydrogel scaffolds were harvested by mechanical disruption with a micropipette until single cells were obtained. Cells were subsequently centrifuged at $400 \mathrm{x} g$ for $5 \mathrm{~min}$ at $4^{\circ} \mathrm{C}$, and the supernatant was removed, rinsed with LG-DMEM and centrifuged again under the same conditions. Total RNA was extracted from culture cells using an RNAiso Plus reagent (Takara Biotechnology, Co., Ltd., Dalian, China) following the manufacturer's protocol, and absorbance was measured at a wavelength of $260 \mathrm{~nm}$ using a spectrophotometer (DU-70; Beckman, Fullerton, CA, USA) to determine the diluted RNA concentration. Each sample $(1 \mu \mathrm{g})$ was reverse transcribed using a PrimeScript ${ }^{\mathrm{TM}} \mathrm{RT}$ reagent kit with gDNA Eraser (Takara Biotechnology Co., Ltd.). qPCR reactions were performed using an Applied Biosystems 7500 Fast Real-Time PCR system (Applied Biosystems; Thermo Fisher Scientific, Inc.) and SYBR ${ }^{\circledR}$ Premix Ex Taq ${ }^{\mathrm{TM}}$ II reagent (Takara Biotechnology Co., Ltd.). Equal quantities of cDNA and specific primers were added to the mix, and the following cycle parameters of PCR was used: Initial denaturation at $94^{\circ} \mathrm{C}$ for $30 \mathrm{sec}$, followed by 40 cycles of denaturation at $94^{\circ} \mathrm{C}$ for $5 \mathrm{sec}$, annealing at $60^{\circ} \mathrm{C}$ for $15 \mathrm{sec}$ and extension at $72^{\circ} \mathrm{C}$ for $10 \mathrm{sec}$. The sequences of forward and reverse primers used for collagen type II $\alpha 1$ (col2 $\alpha 1$; collagen II gene), SOX9, collagen type I $\alpha 1$ (col1 $\alpha 1$; collagen I gene) and collagen type $\mathrm{X} \alpha 1$ (col10 $\alpha 1$; collagen $\mathrm{x}$ gene) are listed in Table I. As described previously (11), mRNA expression levels were analyzed by the $2^{-\Delta \Delta \mathrm{Cq}}$ method using the housekeeping gene glyceraldehyde-3-phosphate dehydrogenase (GAPDH) as an internal control.
ALP activity. ALP is one of the most common indicators of hypertrophic differentiation, and was measured in culture supernatants as described previously (11). At 7, 14 and 21 days, the medium was replaced with phenol red-free DMEM supplemented with $10 \%$ fetal bovine serum, $10 \mathrm{U} / \mathrm{ml}$ penicillin G and $10 \mathrm{mg} / \mathrm{ml}$ streptomycin (GE Healthcare Life Sciences). Supernatants was collected after 1 day and centrifuged at $1,400 \times g$ for $10 \mathrm{~min}$ at $4^{\circ} \mathrm{C}$ to remove particles. ALP activity was immediately assayed using a commercial ALP kit (Nanjing Jiancheng Bioengineering Institute, Nanjing, China) according to the manufacturer's protocol.

Statistical analysis. All data are expressed as mean \pm standard deviation. The differences between multiple-group comparisons were analyzed by one-way analysis of variance followed by Tukey's post hoc test. $\mathrm{P}<0.05$ was considered to indicate a statistically significant difference. Statistical tests were performed using SPSS software version 16.0 (SPSS, Inc., Chicago, IL, USA).

\section{Results}

Effect of icariin on the biosynthesis of ECM. To evaluate the effect of icariin on BMSC chondrogenesis in self-assembling peptide nanofiber hydrogel scaffolds, immunofluorescence was performed after 7, 14 and 21 days of culture. Collagen II is a primary component of cartilage ECM produced by chondrocytes. At each time point, TGF- $\beta 3$ and TGF- $\beta 3+$ icariin groups were positive for collagen II staining; however, the control medium was negative. Analysis of multiple sections of hydrogels revealed that icariin treatment led to more intense and uniformly distributed staining throughout the hydrogel scaffold at day 7 to 21 compared with the TGF- $\beta 3$ group (Fig. 1A). Notably, staining was concentrated to the cell clusters (red arrows), while little staining was detected in single cells (white arrows).

SOX9 is considered to be an early chondrogenic marker and serves a pivotal role in MSC chondrogenesis. The present study stained hydrogel scaffolds with a SOX9-specific antibody after 7 days in culture, and the results demonstrated that cells cultured with TGF- $\beta 3+$ icariin medium exhibited markedly increased positive staining compared with cells in TGF- $\beta 3$ only medium, and no staining was present in control medium (Fig. 1B).

Toluidine blue staining of day 21 hydrogels (Fig. 1C) was consistent with the collagen II staining (Fig. 1A), demonstrating that the cells were enclosed in a metachromatic matrix. The TGF- $\beta 3$ + icariin group had more proteoglycan deposition compared with the TGF- $\beta 3$ group, which was reflected by more intense staining. In addition, chondrocyte-like rounded cells were observed in the TGF- $\beta 3+$ icariin and TGF- $\beta 3$ groups.

Effect of icariin on expression levels of chondrogenesis-specific genes. To further assess chondrogenesis of BMSCs in self-assembling peptide nanofiber hydrogel scaffolds at a genetic level, mRNA expression levels of the chondrogenesis-specific genes $\operatorname{col} 2 \alpha 1$ and SOX9 were determined using RT-qPCR. Compared with the control group, the expression levels of $\operatorname{col} 2 \alpha 1$ and SOX9 mRNA continued to significantly increase from days 7 to 21 in the TGF- $\beta 3$ 
Table I. Primer sequences.

\begin{tabular}{lll}
\hline Gene & \multicolumn{1}{c}{ Forward (5'-3') } & Reverse $\left(5^{\prime}-3^{\prime}\right)$ \\
\hline Col $2 \alpha 1^{\mathrm{a}}$ & CGCCACGGTCCTACAATGTC & GTCACCTCTGGGTCCTTGTTCAC \\
SOX9 ${ }^{\mathrm{a}}$ & GCAGAGACTGAAGACCCTACACAGA & GAGGCAACTTCACGCTGCAA \\
Col1 $\alpha 1^{\mathrm{b}}$ & GCCTCCCAGAACATCACCTA & GCAGGGACTTCTTGAGGTTG \\
Col10 $1^{\mathrm{b}}$ & GCCAGGACCTCCAGGACTATCA & CCCAATGTCTCCTTTCGGTCCA \\
GAPDH $^{\mathrm{c}}$ & TATGACTCTACCCACGGCAA & \\
\hline
\end{tabular}

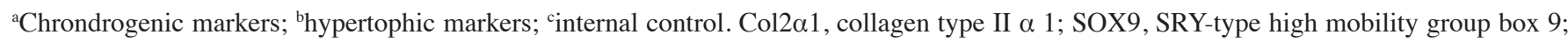
Col1 $\alpha 1$, collagen type I $\alpha 1$; Col10 $\alpha 1$, collagen type $\mathrm{X} \alpha 1$.

\section{A Collagen II}

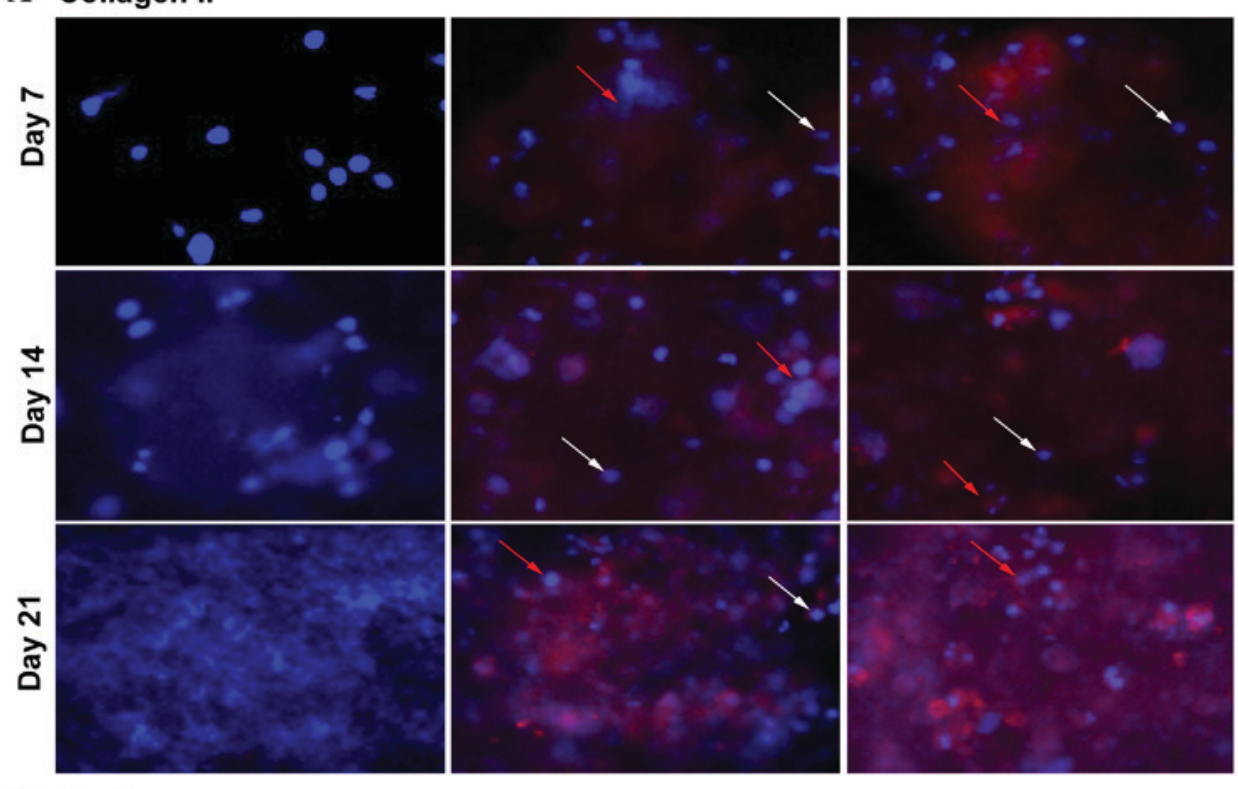

B Sox 9

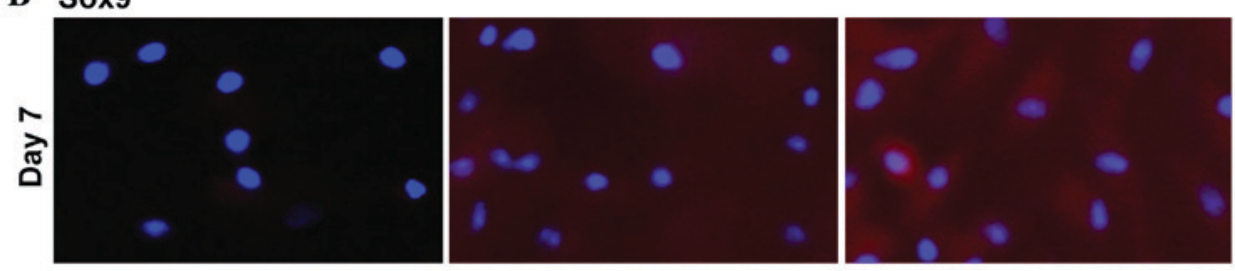

C Toluidine blue

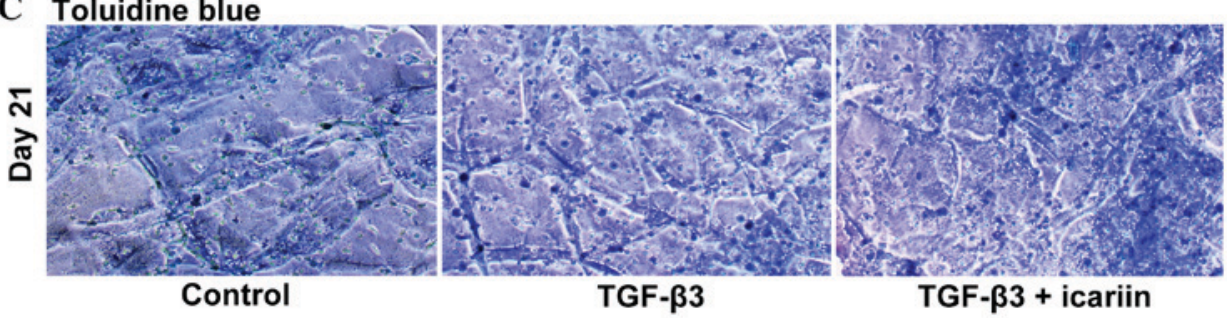

Figure 1. Effect of icariin on the biosynthesis of the extracellular matrix, as detected by immunofluorescence and toluidine blue staining. (A) Cells were stained for collagen II (red) and nuclei were stained with DAPI (blue) after 7, 14 and 21 days in culture. The red arrows indicate collagen II staining in cell clusters, and white arrows indicate staining in single cells. (magnification, x100). (B) Cells were stained for SOX9 (red) after 7 days in culture (magnification, x100). (C) Cells were stained with toluidine blue dye solution after 21 days in culture (magnification, $\mathrm{x} 40$ ). SOX9, SRY-type high mobility group box 9; TGF- $\beta 3$, transforming growth factor- $\beta 3$.

and TGF- $\beta 3+$ icariin groups. Compared with the TGF- $\beta 3$ group, col $2 \alpha 1$ gene expression in the TGF- $\beta 3+$ icariin group was increased by 1.80 -fold $(\mathrm{P}<0.01), 1.60$-fold $(\mathrm{P}<0.05)$ and 1.20-fold ( $\mathrm{P}>0.05)$ at days 7, 14 and 21, respectively (Fig. 2A). Consistent with the expression levels of col $2 \alpha 1 \mathrm{mRNA}$, icariin treatment led to a 1.65-, 1.56- and 1.31-fold increase in SOX9 
A

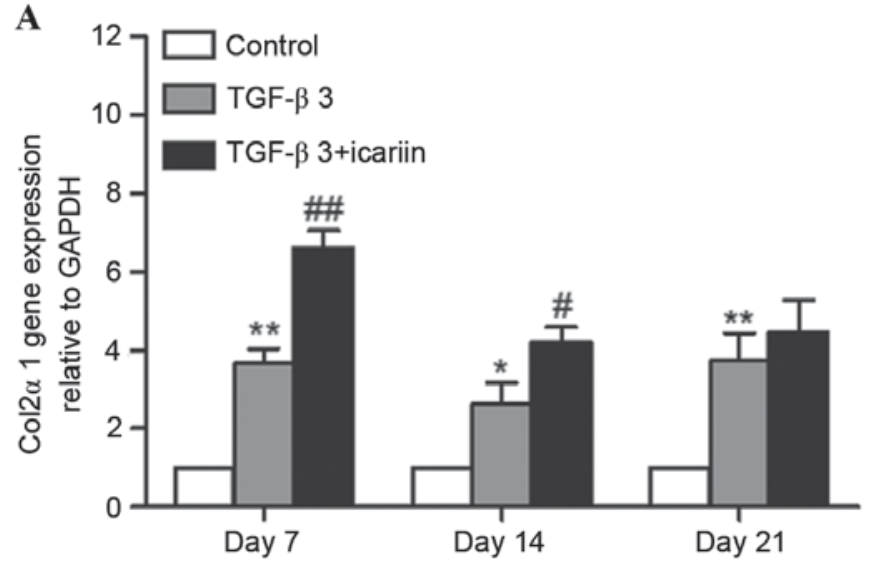

B

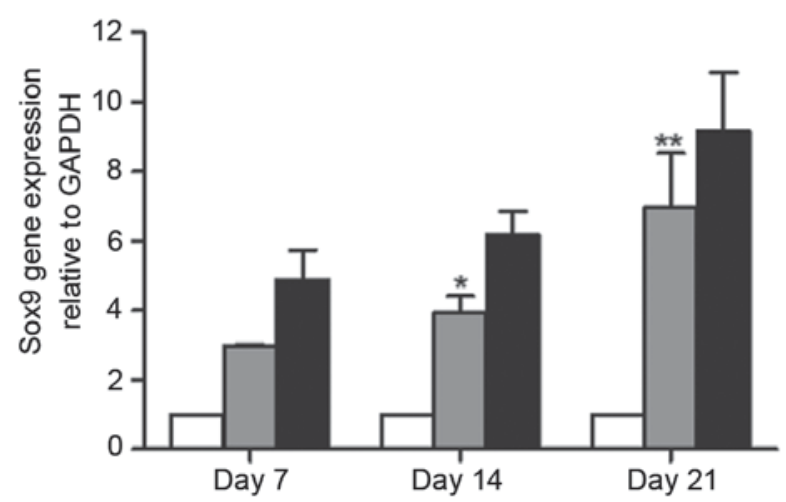

Figure 2. Effect of icariin on gene expression levels of cartilage-specific markers were analyzed by reverse transcription-quantitative polymerase chain reaction after 7, 14 and 21 days in culture. (A) Col2 $\alpha 1$ and (B) SOX9 gene mRNA expression levels. GAPDH served as an internal control. ${ }^{*} \mathrm{P}<0.05$ and ${ }^{* *} \mathrm{P}<0.01$ vs. control group; ${ }^{\#} \mathrm{P}<0.05$ and ${ }^{\# \#} \mathrm{P}<0.01$ vs. TGF- $\beta 3$ group. Col $2 \alpha 1$, collagen type II $\alpha 1$; SOX9, SRY-type high mobility group box 9 ; TGF- $\beta 3$, transforming growth factor- $\beta 3$.

expression levels at days 7, 14 and 21 in comparison with the TGF- $\beta 3$ group (Fig. 2B).

Effect of icariin on cell morphology during chondrogenesis. Due to the transparency of the self-assembled peptide nanofiber scaffold, the morphology of BMSCs encapsulated in the scaffold during chondrogenesis was easily observed. As presented in Fig. 3, spherical, isolated and uniformly seeded cells were observed prior to chondrogenic induction at day 0 . In the TGF- $\beta 3$ and TGF- $\beta 3$ + icariin groups, BMSCs became elongated with long processes and cell-cell contacts with a clustered morphology at day 3 , and the shape of BMSCs further altered from clusters to fibroblasts, and exhibited a spread morphology at day 7. In the control medium, cells maintained the same spherical, isolated morphology as at day 0. Despite these early differences, cells cultured in TGF- $\beta 3+$ icariin medium had a more chondrocyte-like rounded morphology compared with the TGF- $\beta 3$ only group at day 21 , whereas a fibroblastic morphology was visible in the TGF- $\beta 3$ group.

Effect of icariin on hypertrophic differentiation. The present study evaluated the hypertrophic differentiation markers, collagen $\mathrm{x}$ (col10 $\alpha 1$ gene), ALP and collagen I (col1 $\alpha 1$ gene), using immunofluorescence and RT-qPCR analysis. After 21 days of culture, compared with the control group (Fig. 4A), which had no collagen $\mathrm{x}$ staining, the TGF- $\beta 3$ group revealed strong staining (Fig. 4B). However, treatment with TGF- $\beta 3+$ icariin (Fig. 4C) did not lead to more intense staining compared with the TGF- $\beta 3$ group, which was consistent with the results of col10 $\alpha 1$ mRNA expression levels (Fig. 4D). ALP activity was additionally quantified in supernatants of cell culture. Similar to the results observed for collagen $\mathrm{x}$, the group treated with TGF- $\beta 3$ at day 7 exhibited significantly increased ALP activity compared with the control group, whereas a reduction in ALP activity was detected in the TGF- $\beta 3$ + icariin group compared with the TGF- $\beta 3$ group (Fig. 4E). Collagen I was considered as a hypertrophic differentiation and dedifferentiation marker of chondrocytes (22). qPCR analysis revealed that the expression levels of col $1 \alpha 1$ in the TGF- $\beta 3$ group were increased significantly at days 14 and 21 compared with the

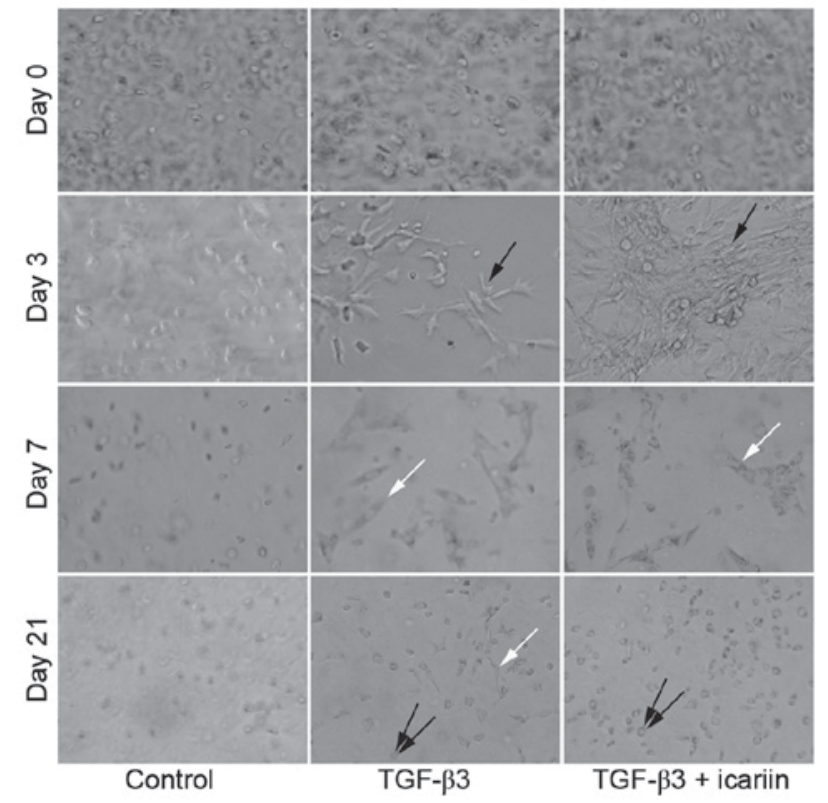

Figure 3. Effect of icariin on cell morphology during chondrogenic differentiation at days $0,3,7$ and 21. Black arrows indicate cell-cell contacts with a clustered morphology, white arrows indicate fibroblastic spread morphology and double black arrows indicate chondrocyte-like rounded morphology. Magnification, $x 100$. TGF- $\beta 3$, transforming growth factor- $\beta 3$.

untreated controls; however, the mRNA levels were decreased in the TGF- $\beta 3$ + icariin group compared with the TGF- $\beta 3$ group, although this difference was not significant (Fig. 4F).

\section{Discussion}

Based on our previous findings that icariin promoted chondrogenic differentiation in traditional monolayer 2D culture (11), the present study hypothesized that icariin may improve cartilage ECM production and reduce hypertrophic differentiation in 3D scaffolds seeded with BMSCs. To maintain a chondrocyte phenotype in monolayer traditional cultures and promote chondrogenesis, various scaffolds chemically conjugated with icariin have previously been used for cartilage tissue 
A Collagen $x$

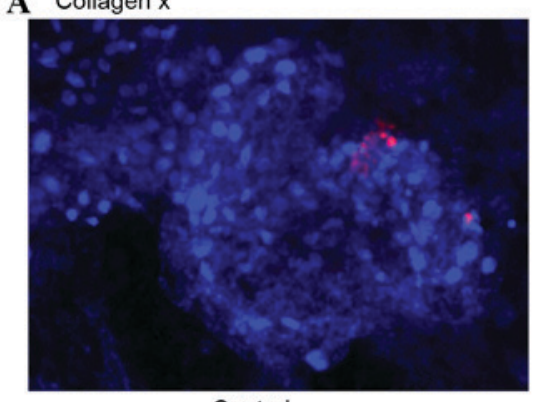

D

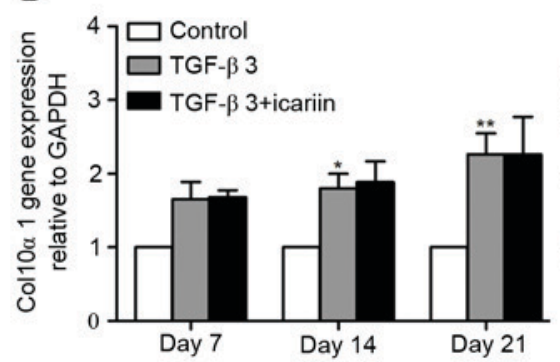

B

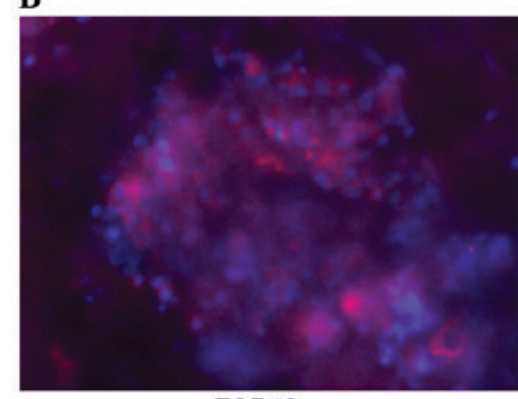

TGF- $\beta 3$

E

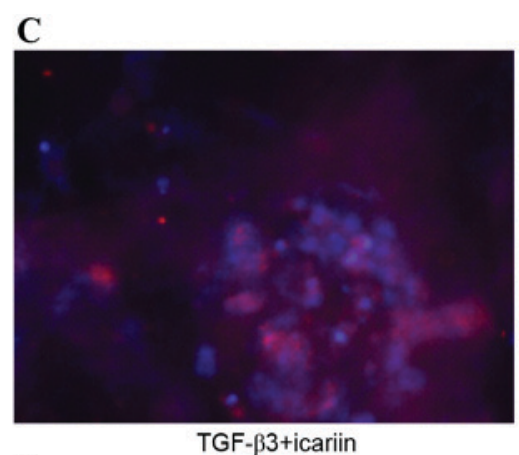

F
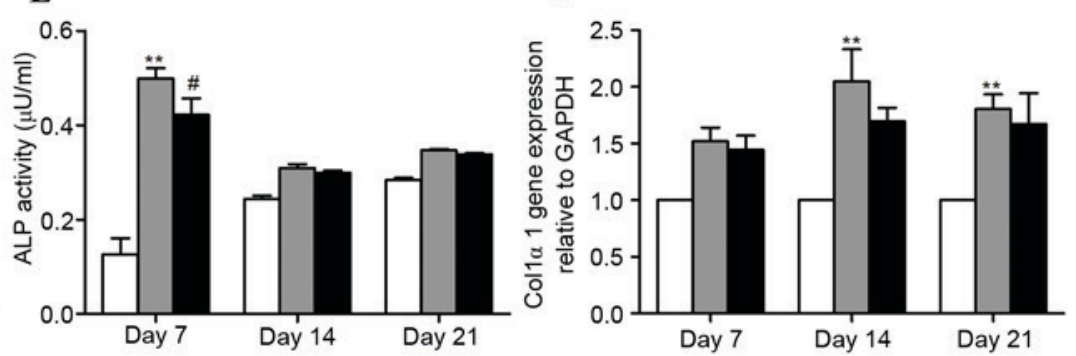

Figure 4. Effect of icariin on hypertrophic differentiation markers. Collagen $\mathrm{x}$ was detected by immunofluorescence in (A) control, (B) TGF- $\beta 3$-treated and (C) TGF- $\beta 3$ + icariin-treated cells (magnification, x100). (D) Col10 $\alpha 1$ gene expression levels were analyzed by RT-qPCR. (E) Soluble ALP activity was quantified in culture supernatants. (F) Coll $\alpha 1$ gene expression levels were analyzed by RT-qPCR. ${ }^{* *} \mathrm{P}<0.01$ vs. control; ${ }^{*} \mathrm{P}<0.05$ vs. control; ${ }^{\#} \mathrm{P}<0.05$ vs. TGF- $\beta 3$. RT-qPCR, reverse transcription-quantitative polymerase chain reaction; TGF- $\beta 3$, transforming growth factor- $\beta 3$; Col10 $\alpha 1$, collagen type X $\alpha$; ALP, alkaline phosphatase; Coll $\alpha 1$, collagen type I $\alpha 1$.

engineering, including hyaluronic acid-icariin/collagen hydrogels (23), hyaluronic acid-icariin conjugate hydrogels (24) and gelatin/hyaluronic acid-icariin composite microspheres (25). Among these biomaterials, self-assembling peptide nanofiber hydrogels are a relatively new scaffold and have emerged as a promising cartilage tissue engineering scaffold for simultaneous cell growth and drug delivery (21). As it is easily loaded with cells, growth factors and drugs, the present study examined the hypothesis by adding icariin and TGF- $\beta 3$ into self-assembling peptide nanofiber hydrogel scaffolds to induce chondrogenic differentiation, and subsequently comparing ECM biosynthesis, gene expression levels and cell morphology. The results of the present study demonstrated that icariin promotes production of ECM components including collagen II protein, and additionally enhances chondrogenesis-specific genes mRNA expression levels, including col $2 \alpha 1$ and the early chondrogenic marker, SOX9.

Consistent with these results, Li et al (7) added icariin into chondrocyte-hydrogel scaffolds and reported that icariin markedly upregulated cartilage-specific gene expression levels of seeded chondrocytes, and accelerated the formation of cartilage-like tissue. They additionally investigated the effect of icariin on the restoration of osteochondral defects, and observed that icariin improved the restoration efficiency and enhanced the integration of new-formed cartilage with subchondral bone. Similarly, when icariin was chemically conjugated to hyaluronic acid/collagen hydrogel scaffolds, the fixed icariin was gradually released, effectively maintaining chondrocyte morphology, promoting cartilage matrix synthesis and forming improved new cartilage tissue (23). Others have investigated the effects of icariin on chondrgogenesis for either chondrocytes or BMSCs in 2D culture, and have demonstrated that the promotion effects of icariin are consistent with those in $3 \mathrm{D}$ culture $(6,11)$. Therefore, these results suggested that icariin promotes the growth of neocartilage and may be a substitute for the use of certain growth factors in cartilage tissue engineering.

Self-assembling peptide nanofiber hydrogel scaffolds are transparent, allowing cells to be observed directly and clearly. In the present study, BMSC morphology began to alter clearly at day 3 , and cell-cell contacts with a clustered morphology were observed in the TGF- $\beta 3$ and TGF- $\beta 3+$ icariin groups. At day 7, the shape of BMSCs further altered from clusters to fibroblasts, exhibiting a spread morphology, and subsequently altered to a chondrocyte-like rounded morphology at day 21, whereas cells maintained the same spherical, isolated morphology in the control medium. The morphological events during chondrogenesis were consistent with numerous previous reports that BMSCs encapsulate in self-assembling peptides (15), pellet culture systems (26) and collagen gel scaffolds (27). Particularly, the aggregation phase was commonly observed in monolayer $2 \mathrm{D}$ cultures and scaffold $3 \mathrm{D}$ cultures $(11,15,27)$. Ichinose et al $(27)$ demonstrated that aggregation of chondroprogenitors was the first step for cartilage formation. Kopesky et al (15) reported that cell-cell contact stimulates cell mitotic activity, and subsequently undergo overt chondrogenesis. The present study revealed that staining was concentrated to cell clusters, whereas little staining was detected in single cells. Thus, increased cell-cell contact in the TGF- $\beta 3+$ icariin group compared with the TGF- $\beta 3$ group may be influential in promoting the chondrogenic effect of icariin.

The present study additionally examined hypertrophic differentiation markers and demonstrated that TGF- $\beta 3$ medium increased the mRNA expression levels of col10 $\alpha 1$, and coll $\alpha 1$, and increased the activity of ALP. Previous reports have demonstrated that growth factors enhance chondrogenesis, 
promote formation of cartilage-like tissue, and inevitably upregulate the expression levels of hypertrophic differentiation markers (2-5). The TGF- $\beta 3+$ icariin group exhibited reduced expression levels of these hypertrophic differentiation markers, suggesting that icariin had no promotion effect on hypertrophy. Consistent with these results, Zhang et al (6) and Li et al (7) reported that icariin downregulates coll $\alpha 1$ mRNA expression levels in chondrocytes.

In conclusion, the present study investigated the effects of icariin treatment on BMSC chondrogenic specific gene expression and ECM synthesis, and characterized cell morphology alterations in self-assembling peptide nanofiber hydrogel scaffolds. It was demonstrated that icariin treatment enhanced cartilage ECM synthesis and cartilage-specific genes expression levels in a 3D microenvironment. However, icariin treatment did not promote hypertrophic differentiation, suggesting that it may inhibit growth factor activity, thus preventing further hypertrophic differentiation. Therefore, these results indicated that icariin may be a potential compound useful for cartilage tissue engineering.

\section{Acknowledgements}

The present study was supported by the National Natural Science Foundation of China (grant nos. 81273508 and 81350017).

\section{References}

1. Lubis AM and Lubis VK: Adult bone marrow stem cells in cartilage therapy. Acta Med Indones 44: 62-68, 2012.

2. Giovannini S, Diaz-Romero J, Aigner T, Heini P, Mainil-Varlet P and Nesic D: Micromass co-culture of human articular chondrocytes and human bone marrow mesenchymal stem cells to investigate stable neocartilage tissue formation in vitro. Eur Cell Mater 20: 245-259, 2010.

3. Aung A, Gupta G, Majid G and Varghese S: Osteoarthritic chondrocyte-secreted morphogens induce chondrogenic differentiation of human mesenchymal stem cells. Arthritis Rheum 63 : 148-158, 2011.

4. Pelttari K, Winter A, Steck E, Goetzke K, Hennig T, Ochs BG Aigner T and Richter W: Premature induction of hypertrophy during in vitro chondrogenesis of human mesenchymal stem cells correlates with calcification and vascular invasion after ectopic transplantation in SCID mice. Arthritis Rheum 54: 3254-3266, 2006.

5. Mueller MB, Fischer M, Zellner J, Berner A, Dienstknecht T, Kujat R, Prantl L, Nerlich M, Tuan RS and Angele P: Effect of parathyroid hormone-related protein in an in vitro hypertrophy model for mesenchymal stem cell chondrogenesis. Int Orthop 37: 945-951, 2013.

6. Zhang L, Zhang X, Li KF, Li DX, Xiao YM, Fan YJ and Zhang XD: Icariin promotes extracellular matrix synthesis and gene expression of chondrocytes in vitro. Phytother Res 26: 1385-1392, 2012.

7. Li D, Yuan T, Zhang X, Xiao Y, Wang R, Fan Y and Zhang X: Icariin: A potential promoting compound for cartilage tissue engineering. Osteoarthritis Cartilage 20: 1647-1656, 2012.

8. Fan JJ, Cao LG, Wu T, Wang DX, Jin D, Jiang S, Zhang ZY, Bi L and Pei GX: The dose-effect of icariin on the proliferation and osteogenic differentiation of human bone mesenchymal stem cells. Molecules 16: 10123-10133, 2011.

9. Liu MH, Sun JS, Tsai SW, Sheu SY and Chen MH: Icariin protects murine chondrocytes from lipopolysaccharide-induced inflammatory responses and extracellular matrix degradation. Nutr Res 30: 57-65, 2010.

10. Sun P, Liu Y, Deng X, Yu C, Dai N, Yuan X, Chen L, Yu S, Si W, Wang X, et al: An inhibitor of cathepsin K, icariin suppresses cartilage and bone degradation in mice of collagen-induced arthritis. Phytomedicine 20: 975-979, 2013.
11. Wang ZC, Sun HJ, Li KH, Fu C and Liu MZ: Icariin promotes directed chondrogenic differentiation of bone marrow mesenchymal stem cells but not hypertrophy in vitro. Exp Ther Med 8: 1528-1534, 2014

12. Li J, Mareddy S, Tan DM, Crawford R, Long X, Miao X and Xiao Y: A minimal common osteochondrocytic differentiation medium for the osteogenic and chondrogenic differentiation of bone marrow stromal cells in the construction of osteochondral graft. Tissue Eng Part A 15: 2481-2490, 2009.

13. Caron MM, Emans PJ, Coolsen MM, Voss L, Surtel DA, Cremers A, van Rhijn LW and Welting TJ: Redifferentiation of dedifferentiated human articular chondrocytes: Comparison of 2D and 3D cultures. Osteoarthritis Cartilage 20: 1170-1178, 2012.

14. Kisiday J, Jin M, Kurz B, Hung H, Semino C, Zhang S and Grodzinsky AJ: Self-assembling peptide hydrogel fosters chondrocyte extracellular matrix production and cell division: Implications for cartilage tissue repair. Proc Natl Acad Sci USA 99: 9996-10001, 2002.

15. Kopesky PW, Vanderploeg EJ, Sandy JS, Kurz B and Grodzinsky AJ: Self-assembling peptide hydrogels modulate in vitro chondrogenesis of bovine bone marrow stromal cells. Tissue Eng Part A 16: 465-477, 2010.

16. Wang B, Sun C, Shao Z, Yang S, Che B, Wu Q and Liu J: Designer self-assembling Peptide nanofiber scaffolds containing link protein $\mathrm{N}$-terminal peptide induce chondrogenesis of rabbit bone marrow stem cells. Biomed Res Int 2014: 421954, 2014.

17. Erickson IE, Huang AH, Chung C, Li RT, Burdick JA and Mauck RL: Differential maturation and structure-function relationships in mesenchymal stem cell- and chondrocyte-seeded hydrogels. Tissue Eng Part A 15: 1041-1052, 2009.

18. Kisiday JD, Kopesky PW, Evans CH, Grodzinsky AJ, McIlwraith CW and Frisbie DD: Evaluation of adult equine bone marrow- and adipose-derived progenitor cell chondrogenesis in hydrogel cultures. J Orthop Res 26: 322-331, 2008.

19. Miller RE, Grodzinsky AJ, Vanderploeg EJ, Lee C, Ferris DJ, Barrett MF, Kisiday JD and Frisbie DD: Effect of self-assembling peptide, chondrogenic factors, and bone marrow-derived stromal cells on osteochondral repair. Osteoarthritis Cartilage 18: 1608-1619, 2010.

20. Miller RE, Grodzinsky AJ, Barrett MF, Hung HH, Frank EH, Werpy NM, McIlwraith CW and Frisbie DD: Effects of the combination of microf racture and self-assembling Peptide filling on the repair of a clinically relevant trochlear defect in an equine model. J Bone Joint Surg Am 96: 1601-1609, 2014.

21. He B, Yuan X, Zhou A, Zhang H and Jiang D: Designer functionalised self-assembling peptide nanofibre scaffolds for cartilage tissue engineering. Expert Rev Mol Med 16: e12, 2014.

22. Cooke ME, Allon AA, Cheng T, Kuo AC, Kim HT, Vail TP, Marcucio RS, Schneider RA, Lotz JC and Alliston T: Structured three-dimensional co-culture of mesenchymal stem cells with chondrocytes promotes chondrogenic differentiation without hypertrophy. Osteoarthritis Cartilage 19: 1210-1218, 2011.

23. Yuan T, He L, Yang J, Zhang L, Xiao Y, Fan Y and Zhang X: Conjugated icariin promotes tissue-engineered cartilage formation in hyaluronic acid/collagen hydrogel. Process Biochemistry 50: 2242-2250, 2015.

24. He L, Yang J, Lu J, Xiao Y, Fan Y and Zhang X: Preparation and characterization of a novel hyaluronic acid-icariin conjugate hydrogel. Materials Lett 136: 41-44, 2014.

25. Yan H,Zhou Z, Huang T, Peng C, Liu Q, Zhou H, Zeng W, Liu L, $\mathrm{Ou} \mathrm{V,} \mathrm{He} \mathrm{S} \mathrm{and} \mathrm{Huang} \mathrm{H}$ : Controlled release in vitro of icariin from gelatin/hyaluronic acid composite microspheres. Polymer Bulletin 73: 1055-1066, 2016.

26. Ichinose S, Muneta T, Koga H, Segawa Y, Tagami M, Tsuji K and Sekiya I: Morphological differences during in vitro chondrogenesis of bone marrow-, synovium-MSCs, and chondrocytes. Lab Invest 90: 210-221, 2010.

27. Ichinose S, Tagami M, Muneta T, Mukohyama H and Sekiya I: Comparative sequential morphological analyses during in vitro chondrogenesis and osteogenesis of mesenchymal stem cells embedded in collagen gels. Med Mol Morphol 46: 24-33, 2013.

This work is licensed under a Creative Commons Attribution-NonCommercial-NoDerivatives 4.0 International (CC BY-NC-ND 4.0) License. 